\title{
Where are the data?
}

\section{Nature Methods now requires data availability statements to be supplied with research papers.}

As the research community embraces data sharing, academic journals can do their part to help. Starting this month, all research papers accepted for publication in Nature, Nature Methods and 11 other Nature titles will be required to include information on whether and how readers can access underlying data.

These statements will report the availability of the ' $\mathrm{min}$ imal data set' necessary to interpret, replicate and build on the findings reported in the paper. Where applicable, they will include details about publicly archived data sets that have been analyzed or generated during the study. Where restrictions on access are in place-for example, in the case of privacy limitations or third-party controlauthors will be expected to make this clear.

The new policy (full details of which are available at www.go.nature.com/2bf4vqn) builds on our longstanding support for data availability as a condition of publication. It also extends our support for data citation, the practice of citing data sets in reference lists in a similar way to citing papers. Authors are encouraged to cite in the reference list data sets that have digital object identifiers (DOIs) assigned to them.

The introduction of data-availability statements follows a trial that began in March 2016 at five Nature journalsNature Cell Biology, Nature Communications, Nature Geoscience, Nature Neuroscience and Nature Physics. The pilot confirmed that there are differences in the culture of data sharing and access between different disciplines and that the lack of obvious public, community repositories can pose a significant barrier to public data deposition. Nevertheless, even in disciplines that are not yet so able to embrace openness and sharing, there is increasing awareness and appreciation that data deposition can enhance the visibility and reuse of published research and that data citation can increase the recognition of those who create and share data.

This new policy will be implemented across the diverse range of Nature journals by early 2017 . We expect that its implementation will shed more light on the reasons for disciplinary differences in data sharing, identify challenges and help to promote the practice of data sharing more widely.

The push to enhance data sharing is not limited to journals alone. A broad drive across the research, funding and publishing communities is underway to make the availability of research data more transparent. Funders, for example, are also introducing data-availability statements. The seven UK research councils require their grant holders to include these statements, and the US National Institutes of Health is asking researchers to provide management plans for their research data.

We expect that offering consistent information on data availability in our papers will promote data reuse by future researchers. And where public data archiving is a mandatory requirement of journals, there is some evidence that including data-availability statements with persistent links to data in published articles is an effective approach to ensuring public data availability and policy compliance (T.H. Vines et al., FASEB J. 27, 1304-1308, 2013).

This new policy follows our publisher Springer Nature's July 2016 launch of an ambitious project to introduce and standardize research data policies across all of its journals (see www.go.nature.com/2by616x). The project sets out a defined common framework for data policy-which Nature policies align with-that enables different journals to encourage sharing of data in a way that reflects the circumstances of respective specialist communities.

\section{And are the tools available?}

\section{We take the opportunity to remind our readers of the materials sharing policy at Nature Methods.}

In addition to availability of the data generated or analyzed in a paper, the availability of materials and tools is equally critical for transparent and reproducible research. We remind our authors and readers that publishing in Nature Methods, as well as in other Nature journals, comes with the commitment to make tools and materials available to other interested scientists.
Materials and methods availability must be described in the paper; any restrictions must be stated and made clear to the editors at submission (see our full policy). Readers who are not able to obtain materials from authors of Nature Methods papers under reasonable conditions, where restrictions were not mentioned in the paper, may contact the Editor-in-Chief to register their complaint. 